\title{
2.13 RESULTS OF OPTICAL TIMING MEASUREMENTS OF THE CRAB NEBULA PULSAR
}

\author{
C. PAPALIOLIOS and N.P. CARLETON \\ Smithsonian Astrophysical Observatory, and Harvard University \\ and \\ P. HOROWITZ \\ Harvard University
}

\begin{abstract}
Absolute time of arrival measurements were made of the optical pulses from the Crab pulsar between September 1969 and April 1970; they were corrected to the solar system barycentre. The fit to the timing data indicates that the slowdown is due to magnetic dipole radiation, but there are significant deviations indicating the presence of small fluctuations and major jumps. There is no evidence of the quasi-sinusoidal behaviour reported by Arecibo. These measurements allowed an integrated light curve to be constructed with high precision.
\end{abstract}

\section{Introduction}

Absolute time of arrival measurements of the optical pulses from the Crab pulsar NP 0531 have been made from September 1969 to April 1970, using the 61-in. reflector at Harvard's Agassiz Station. The timing accuracy, limited only by the available signal strength and the sky background level, is typically 3-8 $\mu \mathrm{sec}$ after an hour of observation.

The signal was accumulated in the usual way (Papaliolios et al., 1968, Horowitz 1969) with some modifications made to allow for accurate absolute timing information (UTC to nearest $\mu \mathrm{sec}$ ) available from East Coast Loran-C to be inserted into the multichannel analyzer. A system similar to ours has been previously reported (Boynton et al. 1969a).

\section{Analysis and Results}

Each night's data consisting of 4 to 10 individual 10-minute runs are combined to give a precise arrival time at the telescope, for a single optical pulse within the observing period. This site arrival time is reduced to a solar-system-barycenter arrival time (Richards et al., 1970). The secular behavior of the pulsar period between successive observations is sufficiently stable so that each of the above pulses can be numbered unambiguously. The result of this data reduction process is shown in Table I. Included in these calculations are the second order general relativistic effects arising from the earth's eccentric orbit around the sun (Hoffman, 1968; Counselman and Shapiro, 1968).

We next attempt to reduce the contents of Table I to some analytic form in order 1) to display in a more transparent way the behavior of the pulsar, and 2) to gain some insights into the physical processes that determine the observed behavior. 
TABLE I

Pulse arrival times at site and solar system barycenter, and corresponding pulse number

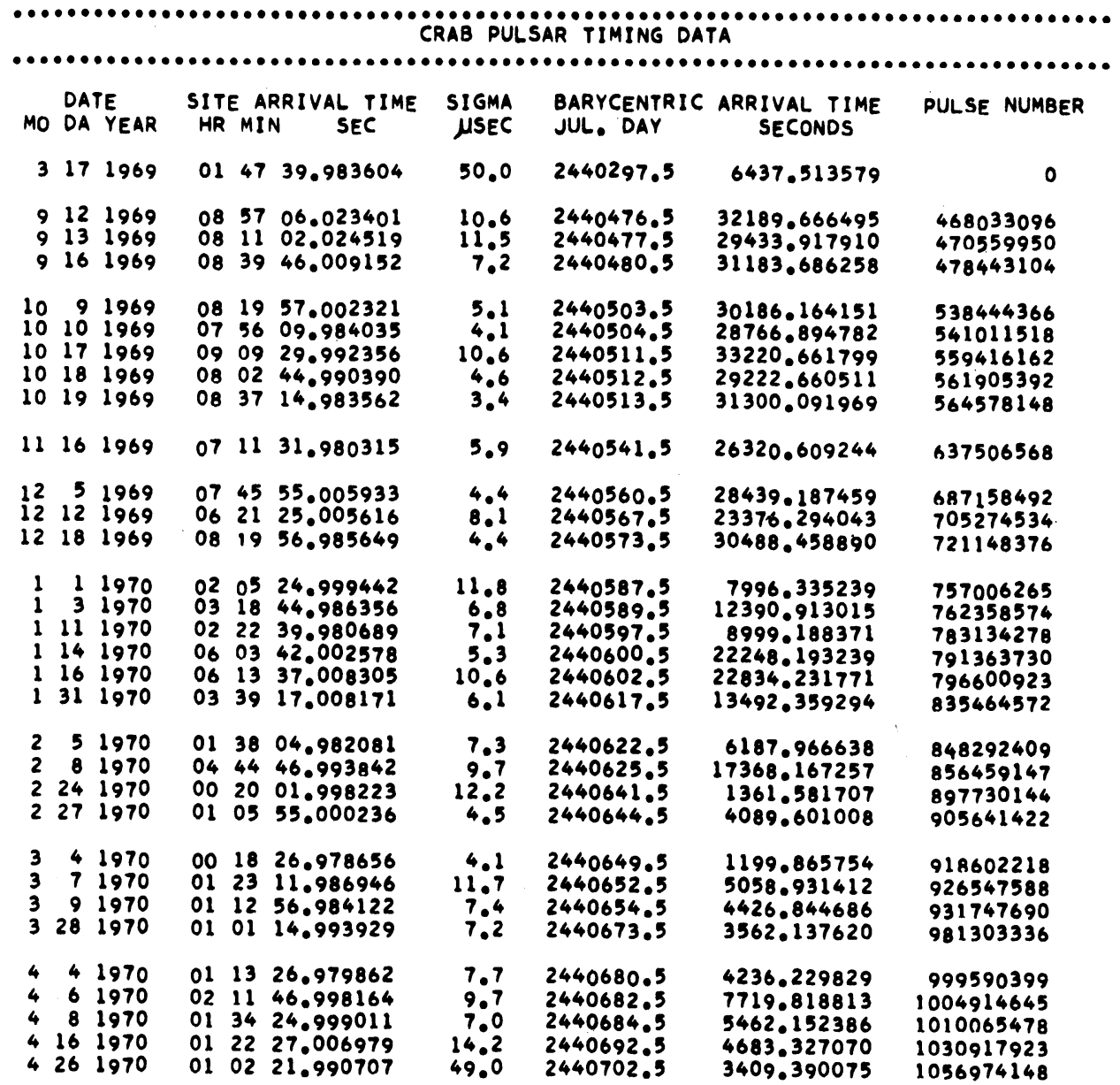

The first relationship between $t$, the barycentric arrival time, and $N$, the pulse number that we try is the truncated power series

$$
\phi=\phi_{0}+f_{0} t+\frac{1}{2} f_{0} t^{2}+\frac{1}{6} \ddot{f}_{0} t^{3}
$$

where we have replaced the integer $N$ by $\phi$, a continuous variable. The parameters $\phi_{0}, f_{0} f_{0}, \ddot{f}_{0}$ are adjusted by the method of least squares to minimize the residuals defined by $r=N-\phi$. If such a fit is performed over the data from March 1969 to the end of September 1969, we have the residuals (converted to units of time) shown in Figure 1. It is clear that for the days not involved in the fit, i.e., past September 1969, the residuals diverge quite markedly. This is not because of the fitting procedure, but because of a sudden increase in the frequency of the pulsar, as others have announced 


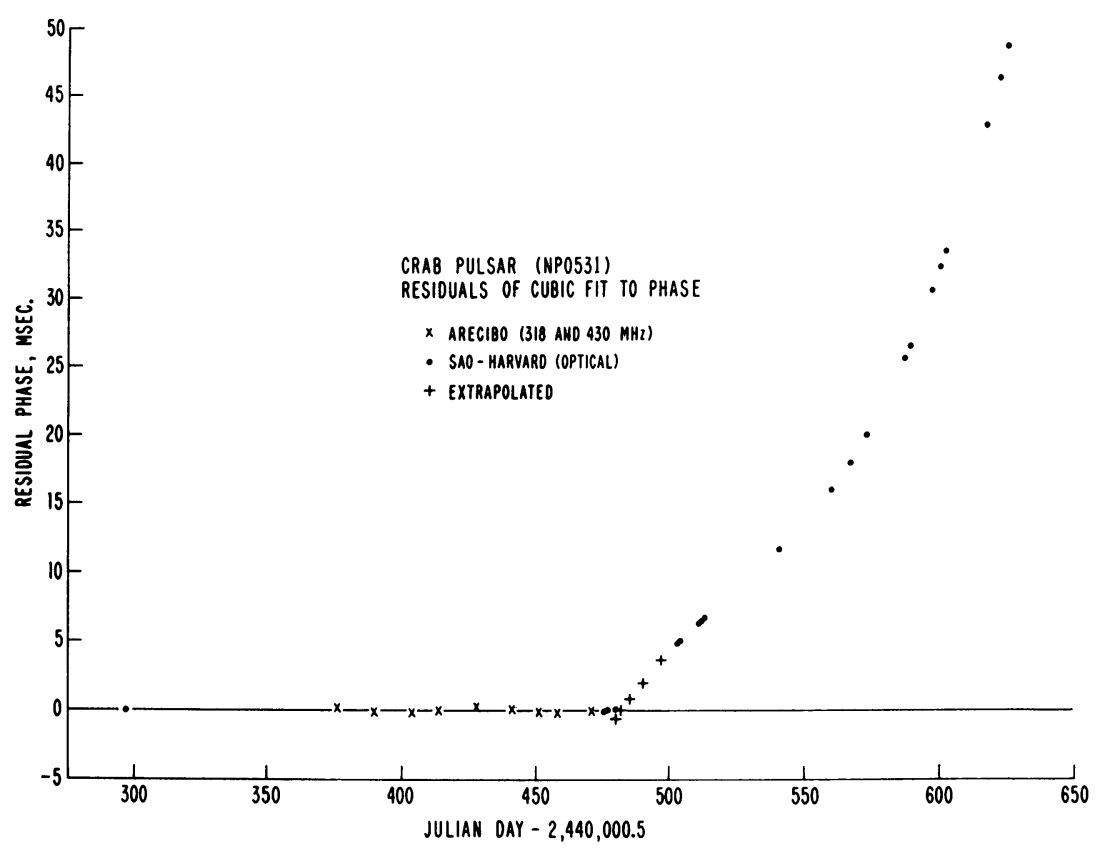

Fig. 1. Residuals vs. Julian day, after weighted arrival times prior to 22 September 1969 (JD 2440484.5) are fitted by equation (1). The phase residuals resulting from this calculation have been converted to units of time.

(Boynton et al., 1969b). A cubic fit to the data past the jump allows us to give the following values to the parameters before the jump, and their changes (final-initial) at the jump,

$$
\begin{array}{ll}
f=30.209297624 \mathrm{~Hz} & \Delta f=9.3 \pm 1 \times 10^{-8} \mathrm{~Hz} \\
\dot{f}=-3.857212 \times 10^{-10} \mathrm{~Hz} / \mathrm{sec} & \Delta \vec{f}=-1.8 \pm .1 \times 10^{-14} \mathrm{~Hz} / \mathrm{sec} \\
\ddot{f}=1.04 \times 10^{-20} \mathrm{~Hz} / \mathrm{sec}^{2} & \Delta \ddot{f}=5.9 \pm 1 \times 10^{-21} \mathrm{~Hz} / \mathrm{sec}^{2} \\
t=\text { JD } 2440484.5 \pm 4=20 \text { September } 1969 \pm 4 \text { days }
\end{array}
$$

If this jump is the result of a sudden readjustment of the equatorial radius of the outside crust, then this radius changed by only $15 \mu$. It can easily be shown that changes of this size should be happening about once a week, but no other has been observed. Another explanation for the jump could lie in Scargle's observation (Scargle and Harlan, 1970) of changes in nebulosity following the jump. A cubic fit to all the data following the jump up to 26 April 1970 results in residuals shown in Figure 2. These residuals, particularly the early ones, are far greater than what one would expect from the measuring process. There is some evidence of a post-jump relaxation lasting up to 1 January 1970 . Using the data past 1 January 1970 results in residuals shown by the solid circles of Figure 2 .

Since the residuals are considerably larger than the measurement errors (which are too small to be shown in Figure 2 but are listed in Table I) we conclude for the present 


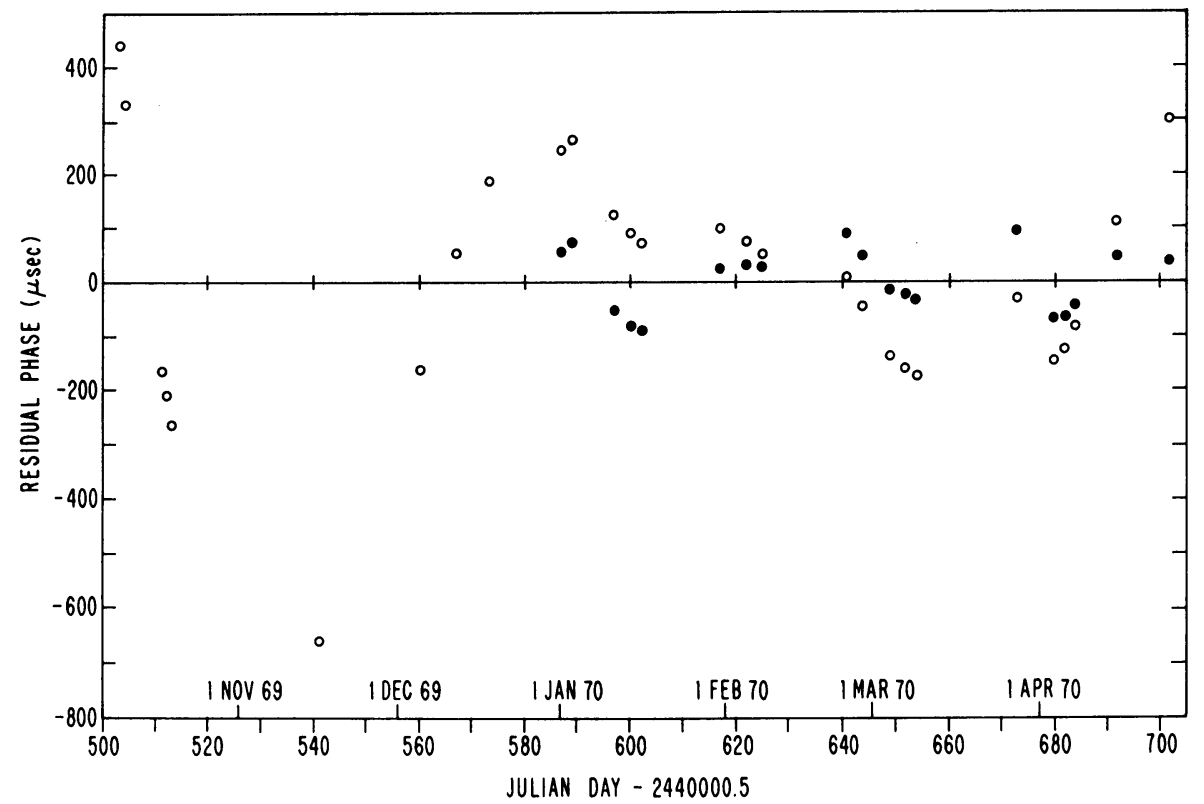

Fig. 2. Residuals $v s$. Julian day; $\bigcirc=$ residuals of a cubic fit from 1 October 1969 to 26 April 1970. $=$ residuals of a cubic fit after 1 January 1970. The braking parameter $n$ is 2.70 and 2.61, respectively, with an uncertainty of about $5 \%$ due to possible errors of a second of arc in the alignment of solar system coordinates relative to NP 0531. The measurement errors are too small to show in this figure but can be obtained from Table I.

that Equation (1) is not the appropriate relationship between $t$ and $\phi$. Modifying Equation (1) by adding a quartic term reduces the residuals somewhat, but this modification is rejected because of the anomalously large contribution to $\phi$ from the additional term.

Another useful relationship is obtained from

$$
f=-a f^{n}
$$

The braking parameter $n$ defined by Equation (2) has the value 3 if the slowdown of a spinning rigid neutron star is due to solely dipole radiation, and has the value 5 for quadrupole radiation. The solution of (2) is

$$
\phi=\phi_{1}+\alpha(1+t / L)^{k}
$$

where $k=(n-2) /(n-1)$, and $L$ is a 'lifetime' parameter.

Least-squares adjustment of the four parameters in equation (3) results in residuals that are essentially identical to the ones obtained from the use of Equation (1). Even such diverse forms as

and

$$
\phi=\phi_{1}+\alpha\{(t+L)+\beta[\ln (t+L)+2]\}^{1 / 2}
$$

$$
\phi=\phi_{1}+\alpha(t+L)^{3 / 4}+\beta(t+L)^{5 / 4}
$$


which are the solutions expected if both dipole and quadrupole radiations are present where dipole radiation is dominant in (4) and quadrupole is dominant in (5), result in residuals identical to those previously obtained.

These results indicate that it is very difficult to compare theoretical explanations with one another, since only the number of adjustable parameters seems to matter. The underlying reason for this unfortunate property appears to be noise, so that all simple relationships between $\phi$ and $t$ will result in excessive residuals.

The braking parameter $n$ varies between 2.6 and 3.7 depending on the string of data one uses in the least-squares fitting process. If we set $n=3$ (the case for pure dipole slowdown) then we see from equation (3) that

$$
t=t_{0}+P_{0} \phi+\frac{1}{2} P_{0}^{\prime} \phi^{2}
$$

This leads to another means of data analysis, the method of divided differences (Buckingham, 1957) which allows us to recognize the existence of small fluctuations

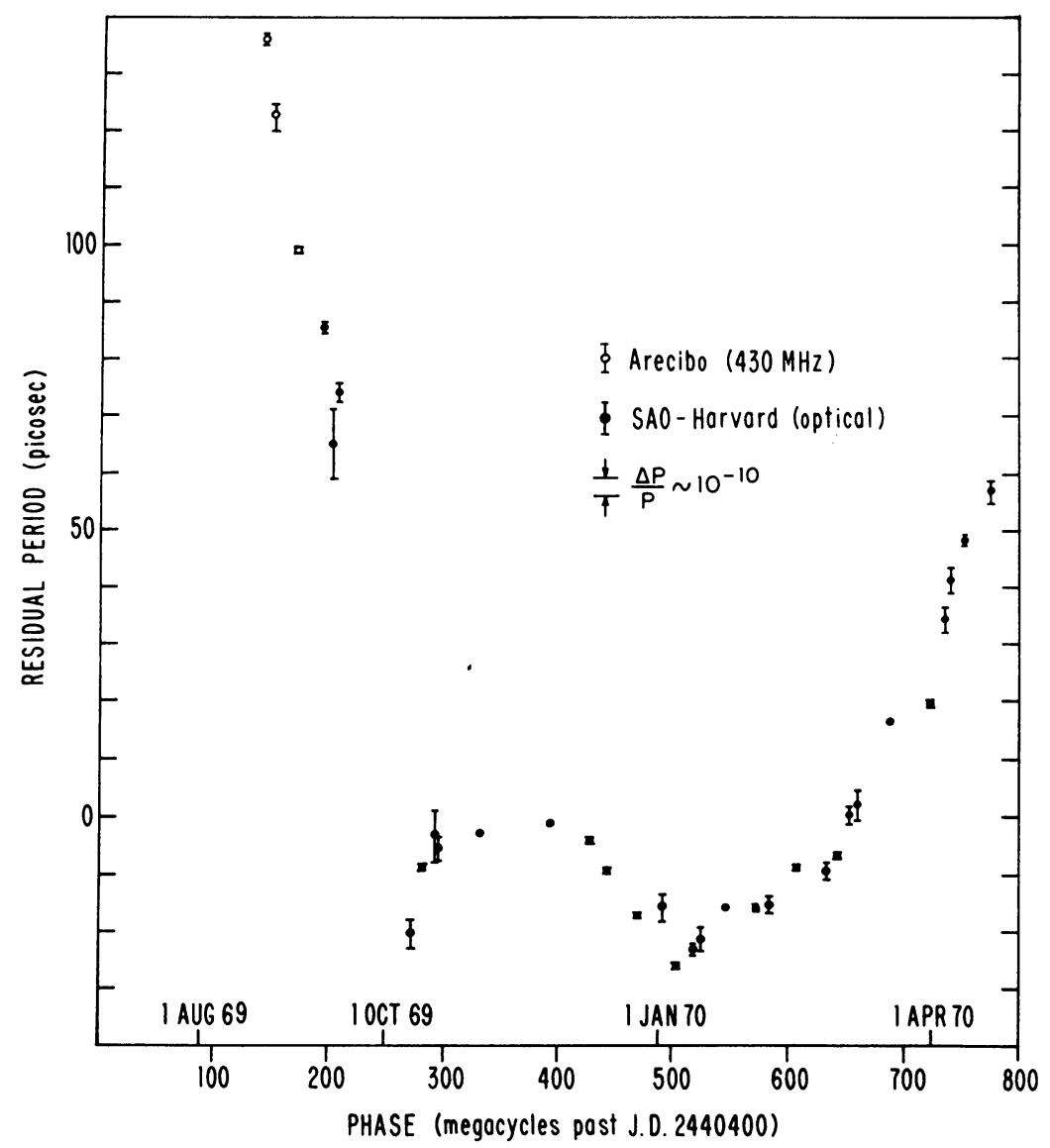

Fig. 3. Residual period vs. phase, calculated by divided differences, with a linear term subtracted. Error bars are omitted when less than 0.35 picosec. 
in the arrival times as well as the major ones such as the jump previously noted. In addition this method is able to suggest interpretations that might not otherwise have been suspected. If we assume that Equation (6) is valid between every pair of measured points $(t, N)$ then at $N=\frac{1}{2}\left(N_{1}+N_{2}\right)$ the period $P$ is exactly $\left(t_{2}-t_{1}\right) /\left(N_{2}-N_{1}\right)$. Equation (6) also implies that the period $P$ is a linear function of $N$ (or $\phi$ ). We perform these calculations, subtract out a linear dependence of $P$ on $\phi$, and plot the remaining residual periods vs. $\phi$ in Figure 3. This graph shows clearly the jump in period that occurred on 20 September 1969, the relaxation following the jump, and the small fluctuations throughout the year. These fluctuations in period of about one part in $10^{10}$ make it difficult to extract from the present timing measurements any useful information on the gravitational red shift, and on the solar system ephemeris. There is also no evidence of a continuation of the sinusoidal oscillation that was reported to exist before the jump by Richards et al. (1970).

If we perform the above analysis on the Arecibo data, kindly supplied to us by the Arecibo group, then the results are as shown in Figure 4. The two straight lines suggest a pure-dipolar slowdown with a single jump in dipole moment. The phase residuals calculated on the basis of this interpretation, which incidentally contains only five adjustable parameters instead of Arecibo's seven, are shown in Figure 5 and are as small as those of the Arecibo group. If our interpretation is correct then there is no sinusoidal oscillation, but instead the dipole moment increased by 3.7 parts in $10^{5}$ on JD 2440410.4 .

Finally, as a simple byproduct of our timing measurements, we can superpose the light curves taken throughout the year, and still maintain the $32-\mu \mathrm{sec}$ resolution. The

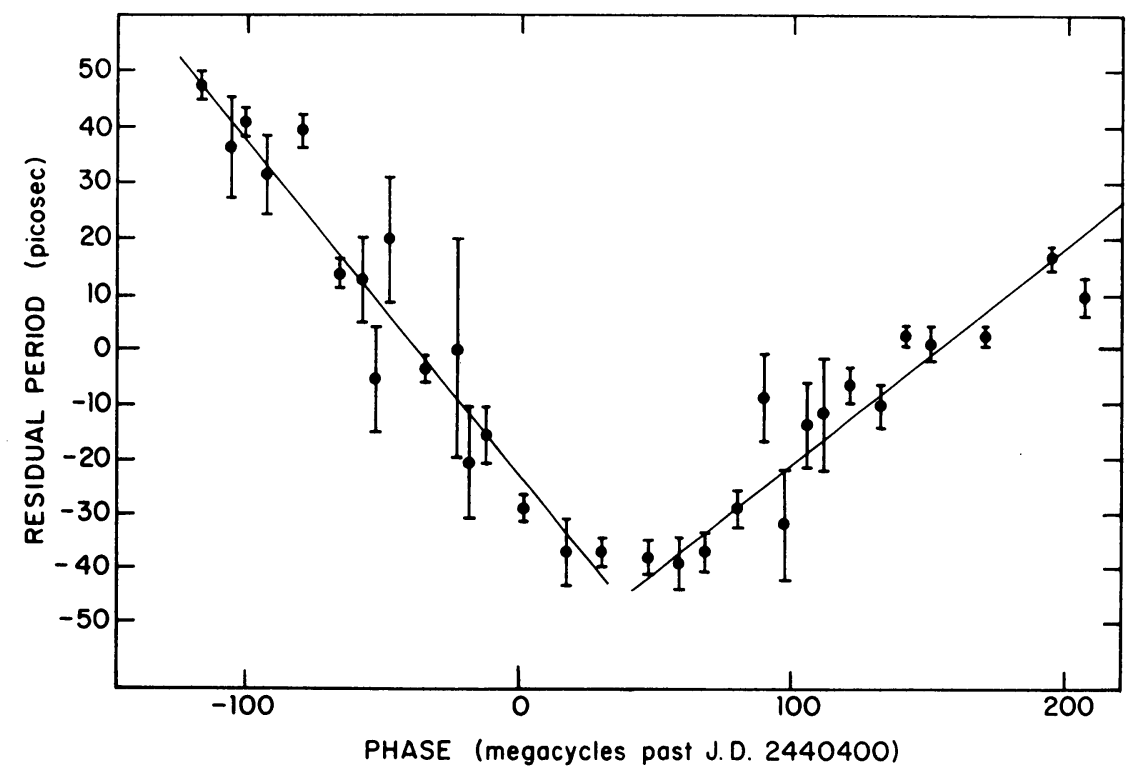

Fig. 4. Residual period vs phase of the summer Arecibo data, with a linear term subtracted. 


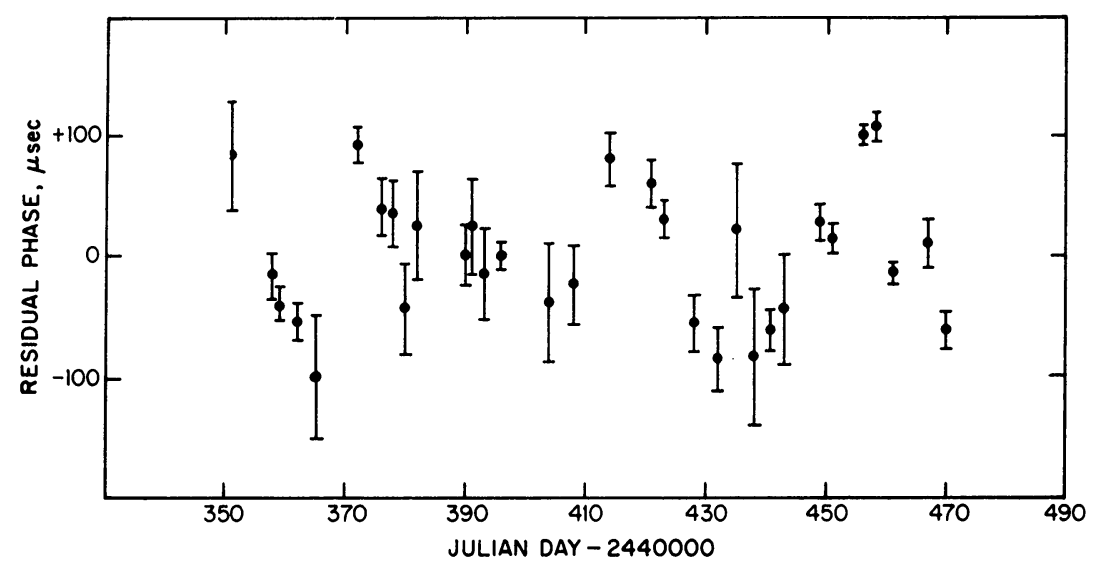

Fig. 5. Residuals $v s$. Julian day, after fitting a dipole braking law with a single jump in the dipole moment to the radiofrequency arrival times obtained during the summer of 1969 by the Arecibo group.

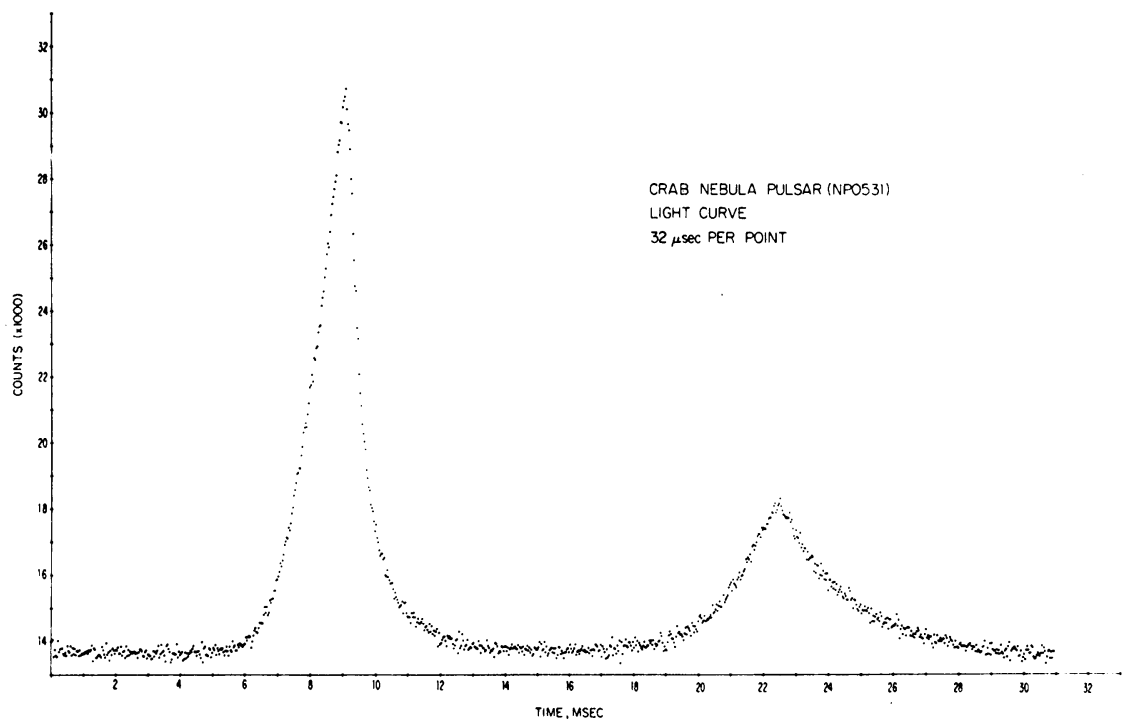

Fig. 6. Superposed light curve from ten nights of observing between October 1969 and March 1970. Total observing time is about ten hours.

result of such a superposition (representing about $10 \mathrm{~h}$ of observation throughout the year) is shown in Figure 6. There is no evidence of rounding at the top of the main peak even down to this short time scale. The time interval of $13.376 \pm 0.032 \mathrm{msec}$ between the peaks of the two light pulses agrees with the $13.36 \pm 0.03 \mathrm{msec}$ interval between the two sharp spikes recorded at $430 \mathrm{MHz}$ by the Arecibo group (Rankin et al., 1970). 


\section{Acknowledgements}

We are very grateful to I. I. Shapiro and C. C. Counselman, III, of MIT, and to F. D. Drake and D. W. Richards of Cornell University for their generous sharing of information, and for many interesting and stimulating conversations. We are similarly grateful to our colleagues G. G. Fazio, L. Goldberg, D. Hearn, B. Kaplan, R. V. Pound, and E. M. Purcell, and members of the Harvard Bubble Chamber Group. Messrs. C. R. H. Tsiang, J. T. West, and W. Wright of the Smithsonian Observatory aided us greatly in our measurements of absolute time, and M. Mattei of Agassiz Station was a valuable assistant in the observing. One of us (P. H.) is grateful to the Society of Fellows for support.

\section{References}

Boynton, P. E., Groth, E. J., III, Partridge, R. B., and Wilkinson, D. T.: 1969a, Astrophys. J. Letters 157, L197.

Boynton, P. E., Groth, E. J., III, Partridge, R. B., and Wilkinson, D. T.: 1969b, IAU Circ. No. 2179.

Buckingham, R. A.: 1957, Numerical Methods, Pitman, London, p. 92.

Counselman, C. C., III, and Shapiro, I. I.: 1968, Science 162, 352.

Hoffman, B.: 1968, Nature 218, 667.

Horowitz, P.: 1969, Rev. Sci. Instr. 40, 369.

Papaliolios, C., Carleton, N. P., Horowitz, P., and Liller, W.: 1968, Science 160, 1104.

Rankin, J. M., Comella, J. M., Craft, H. D. Jr., Richards, D. W., Campbell, D. B., and Counselman, C. C., III: 1970, Astrophys. J. Letters 160, L1.

Richards, D. W., Pettengill, G. H., Counselman, C. C., III, and Rankin, J. M.: 1970, Astrophys. J. Letters 160, L1.

Scargle, J. D. and Harlan, E. A.: 1970, Astrophys. J. Letters 159, L143.

\section{Discussion}

D. T. Wilkinson: It now is clear that the behaviour of residuals is a strong function of the model chosen and the distribution of the data. We disagree with several of the results of the Harvard group. Our data indicate that the exponential decay of the transient part of the discontinuity has a time constant of about 2 weeks; its effect is essentially gone by 1 December. The value of $Q$, as defined by Pines et al., is about 1 or larger in our fits. The changes in $n$ reported are modelled in our fits as quartic or periodic terms. Finally, we also see the bumps in March and April of 1970, seen by the Lick group. They are unexplained in any models discussed here.

C. Papaliolios: We do observe a different behaviour for the pulsar before about 1 January 1970, and then after that date. This difference is not an artifact of the fit but is clearly indicated by the method of divided differences.

Hills: As a member of the Berkeley group I would like to comment on the apparent differences in the results of the pulse timing presented by the different groups. As far as we know there are no actual contradictions in the arrival times measured but clearly the phenomena which show up depend very much on the details of the observations made. Thus our measurements which have greater timing accuracy ( $<2 \mu \mathrm{sec}$ for a good night) and more frequent observations, but a shorter time base than the others, show only the structure on a scale which is very small (some might say insignificant), whereas the measurements over longer periods with lower resolution show the more dramatic changes. We look forward to combining our data with that of other groups to check our measurements and to try to understand whether the phenomena described can all be considered part of the same behaviour or whether a more complex model must be used.

C. Papaliolios: In studying the slow-down of the pulsar, i.e., the braking parameter, it is not 
sufficient to consider just the radiation torques. The changes in moment of inertia can also play an important role; after all, we know that the pulsar is slowing down, therefore, its oblateness is also changing. Although this effect is not in itself sufficient to account for the total discrepancy between $n=3$ (magnetic dipole slowdown) and the observed average value of about 2.6, it is in the proper direction. Other effects which should be included in a detailed calculation are the changing dipole moment that comes from the shape changes and the additional effects due to the compressibility of the pulsar. 